\title{
The Inner Pressure of the Atoms in a Solid in Refined TFD Theory
}

\author{
Ru-Zeng Zhu ${ }^{1 *}$, Yu-Hua Wen ${ }^{2}$, Jin Qian ${ }^{1}$
}

1. State Key Laboratory of Nonlinear Mechanics (LNM), Institute of Mechanics, Chinese Academy of Sciences, Beijing 100080, China

2. Department of Physics, Tsinghua University, Beijing 100084, China

\begin{abstract}
The relation between the inner pressure of an atom in a solid and the density of energy of electrons under Refined TFD theory is given.
\end{abstract}

Keywords: Inner pressure, TFD theory, energy density

\section{Introduction}

For the structures and properties of Nano materials, and the behaviors of the Micro and Nano systems, the quantum effects are known of importance more or less. The semi-classical Thomas-Fermi-Dirac theory is a simplified one of those theories to treat of the quantum effects. In TFD theory, there is no quantum tunnel effect, i.e. there is no possibility of electrons being checked up in the area where the potential is bigger than the energy of the electrons. In 1990s, Chengs proposed the refined TFD theory [1-4], in which the quantum tunnel effect for the atoms in solid is included and the tunnel electrons provide a pseupotential for the system. Chengs [5] have shown that the pseupotential will affect the properties of materials, so that to discuss this theory and its deductions in detail may be useful for the study of the structures and properties of Nano materials, and the behaviors of the Micro and Nano systems.

In reference [6], the pressure on the atomic surface in a solid is given by the energy density of electrons and pseupotential under Refined
TFD theory. In the present paper, the inner pressure of an atom in a solid under the same theory is given by the energy density of electrons.

\section{Main idea of derivation}

According to reference [6], consider an atom in a solid in the basic state as a Fermi ball with radius $R$ and with the surface charge density $\sigma=Q / 4 \pi R^{2}$ on its surface $S(R)$, contributed by the tunnel electrons belonging to this atom, where $Q$ is the total charge of the tunnel electrons.

Let $p(r)$ be the pressure at $r$ from the nuclei. To deduct $p(r)$ at $r<R$, we draw a surface $S(r)$ with radius $r$ and the center at the nuclei. The total energy $E$ of the system can be written as

$$
E=E_{\text {in }}+E_{\text {out }}+E_{\text {i-o }}+\Phi,
$$

where $E_{\text {in }}, E_{\text {out }}, E_{t-o}$ and $\Phi$ are respectively the energy of the part inside $S(r)$ including the

\footnotetext{
"Corresponding author. E-mail: zhurzailnmimechacen (Ru-Zeng Zhu).
} 
nuclei, that of the part outside $S(r)$ except the surface charge, the interaction energy between the two parts, and the pseupotential, which includes the self-energy of the surface charge and the interaction energy between it and both of the nuclei and all the volume charges. We have

$$
\begin{aligned}
& E_{\text {m }}=\int_{r_{1}<r}\left(E_{k}+E_{c o n}+E_{c x}\right) d V_{1}, \\
& E_{\text {oul }}=\int_{r<r_{1}<R}\left(E_{k}+E_{c o u t}+E_{e x}\right) d V_{1}, \\
& E_{i-o}=-\int_{r}^{R} \phi\left(r_{1}\right) e n\left(r_{1}\right) d V_{1}, \\
& \Phi=-Q^{2} / 2 R,
\end{aligned}
$$

where $e, \quad n\left(r_{1}\right), E_{k}\left(r_{1}\right), E_{\text {cin }}\left(r_{1}\right), E_{\text {cout }}\left(r_{1}\right)$, $E_{e x}\left(r_{1}\right)$ and $\phi\left(r_{1}\right)$ are the absolute value of the charge of an electron, the density of electrons at a distance $r_{1}$ from the nuclei, the density of kinetic energy of electrons at $r_{1}$ from nuclei, that of Coulomb energy provided by all the charged particles inside $S(r)$, that of Coulomb energy provided by all the charged particles outside $S(r)$ except the surface charge, that of the exchange energy among electrons, and the potential at $r_{1}$ provided by all the charges inside $S(r)$, respectively. Eq.(5) can be found in reference [6], and the expressions of $E_{k}\left(r_{1}\right)$ and $E_{e x}\left(r_{1}\right)$ can be found in reference [7].

In order to get the expression of the inner pressure of an atom under the refined TFD theory, let's reduce $S(r)$ to $S(r+d r)$ slowly with the volume density and surface density of the electrons outside $S(r)$ remaining unchanged, while the electrons inside $S(r)$ changing adiabatically so as to be kept inside $S(r+d r)$. For this process, we have

$$
(d E)_{a d}=-p(r)(d V,(r))_{a d},
$$

where $V,(r)$ is the volume included by $S(r)$ and the subscript ad means adiabatic. Then we can prove that Eq.(6) can be reduced to

$$
p(r)=-\left(d E_{i n} / d V(r)\right)_{a d} .
$$

On the other hand, for basic state we are considering, the energy functional (1) should take the least value under the restriction condition of the total number of the electrons inside the surface $S(R)$

$$
N=\int_{r_{1}<R} n\left(r_{1}\right) d V_{1}
$$

being constant, so that by introducing Lagrangian multiplier $\lambda$, we can prove

$$
\partial\left(E_{k}\left(r_{1}\right)+E_{e x}\left(r_{1}\right)+E_{c i n}\left(r_{1}\right)\right) / \partial n\left(r_{1}\right)=-\lambda .
$$

From Eqs.(2), (7), (14) and the conservation of the number of the electrons inside the surface $S(r)$, we can obtain

$$
p(r)=-\left\{E_{k}(r)+E_{e x}(r)-e n(r) \phi(r)+\lambda n(r)\right\} \text {. }
$$

By substituting the expressions for $E_{k}, E_{c n}$ and $E_{e x}[7]$ into Eq.(9), we can get the expression for $\lambda$ in detail. Substitution of this expression for $\lambda$ into Eq.(10) gives the expression for the inner pressure of the atom in a solid

$$
p(r)=(1 / 3)\left[2 E_{k}(r)+E_{e x}(r)\right] .
$$

\section{Conclusion}

The inner pressure of an atom in a solid under Refined TFD theory is determined only by the energy density of electrons through Eq.(11), although there is a surface charge layer surrounding the atom.

\section{Acknowledgments}

The supports from the National Natural Science Foundation of China (No.10172088) are gratefully acknowledged.

\section{References}

[1] Cheng, K.J., Cheng, S.Y. The application of the TDF model and Yu's theory to material design, Science Nature Progress, 3(5)(1993)417-432 (In Chinese) 
[2] Cheng, S.Y., Cheng, K.J. Computation on heat of formation and EOS of alloy by a refined TFD model, Acta Physica Sinica (Overseas), 2(6)(1993)439-448

[3] Cheng, K.J., Cheng, S.Y. A new method to the calculation of equation of state at null temperature, Proc. of CMRS International Symposoum (1990)783-788

[4] Cheng, K.J., Cheng, S.Y. A new method in the calculation of EOS and the study of phase diagram of materials, Advances in Mechanics, 21(1991)23-30 (In Chinese)

[5] Cheng, K.J., Cheng, S.Y. The effect of minority charges on the characteristics of material, Chinese $J$. of Materials Research, 18(1)(1996) (In Chinese)

[6] Cheng, K.J., Cheng, S.Y. Proof of the validity of the Virial Theorem in the equation of state by the Thomas-Fermi-Dirac model, Acta Physica Sinica (Overseas), 4(5)(1995)344-349

[7] Lundqvist, S., March, N.H. Theory of the inhomogeneous electron gas, New York: Plenum Press, 1983 